\title{
Enumeratio specierum Salviarum ex insulis Honsiu, Sikoku, Kiusiu, Liukiu et Formosa adhuc cognitarum.
}

Auctore

\section{Yushun Kudo.}

Hoc opuscuium est parva pars operis "Revisio Labiatarum Japonicarum" dicti, quod, Professoris J. Matsumura ductu, mense junio anni 1911 in Instituto Botanico Universitatis Tokiensis inceptum, mense junio anni 1912 complevi. Maximas gratias Professori J. Matsumura, ob ejus peritissimum humanissimumque ductum et auxilium reddere debeo.

Tametsi Linnaeus, in prima editione Specierum Plantarum, ${ }^{1}$ septem et viginti Salvias species eo tempore notas descripsit, tamen nullam speciem in terra Japoniae inventam describebat. Prima species quae in terra Japoniae inventa fuit, a THUnBERG, ${ }^{2)}$ anno 1789, descripta est, et "Salvia japonica" nominata. Multi novi scriptores, de illa specie loquendo, in errorem inducti sunt, et hanc speciem cum planta japonice "Akinotamuraso" dicta identicam esse putaverunt. Sed si e tempore florendi judicetur, hanc speciem cum planta japonice "Natsunotamuraso" dicta identicam esse viditur.

MiQUeL $^{3)}$ terrae Japoniae quattour Savlias species enumeravit quae sunt Salvia nipponica, japonica, deversifolia et plebeia. Planta, quaedam, lingua nostra "Akigiri" dicta, primo, ut nova specie, ab illa descripta est, et "Salvia nipponica" apellata. Praetera, Salviam brachiatam (S. plebeiam) et unam novam

1) Linn. Spec. Pl. ed. 1. (1753) p. 23.

2) Thuns. Fl. Jap. (1784) p. 22, t. 5.

3) Miqued, Prol. Fl. Jap. (1866-67) pp. 39, 40. 
speciem enumeravit, quae tamen, "S. diversifolia" dicta, est solum forma quaedam $S$. chinensis.

Anno 1875, auctores Franchet et Savatier,') in primo volumine Enumerationis Plantarum Japonicarum, tres species enumeraverunt. Primo, illi auctores novam varietatem Glabrescentem nominatam descripserunt, et hujus varietatis speciem typicam, etiam in characteribus coloris florum etc. variabilem esse notaverunt. Secundo, de planta ab eis "S. japonica" dicta locuti sunt et hujus quattuor varietates, quarum una est nova, enumeraverunt. Ex illorum investigationibus Miquel's nova species "S. diversifolia" dicta cum una illarum varietate identicam esse putamus. Planta japonice "Harunotamuraso" dicta, ut varietate hujus speciei, sub nomine " $S$. japonica. var. pumila", descripta fuit. Haec species a nobis (Professore J. Matsumura et me ipso) “S. vernalis", ob tempus florendi, apellata est, et a cl. MAKINO "S. Ranzaniana" nominata. In secundo volumine ${ }^{3)}$ ejusdem libri breves diagnoses de S. japonicae varietatibus datae sunt.

Anno 1885, cl. ENGLER, in volumine sexto diarii sui "Botanische Jahrbücher nominati, unam speciem" in Insula Oshima collectam designavit.

Temperibus deversis, Professor J. Matsumura in "Tokyo Botanical Magazine" et aliis operibus, nonnullas Salvias species in Japonia crescentes, descripsit. Anno 1897, unam novam speciem "S. pygmaeam" dictam in "Tokyo Botanical Magazine"5) descripsit; haec species inter saxa ad flumen prope Ooshima collecta fuit et ea subgenus quod Covola dicitur pertinet. Idem auctor unum specimen, quod in provincia Awa collectum erat, diu in herbario suo servavit, et posterius illud sub nomine novo "S. trisecta" produxit. Index completus plantarum spontanearum et introductarum japonicarum Salviarum in opere

1) Fr. et SAv. Enum. Pl. Jap. Vol. I. (1879) pp. 371-5.

2) Makino, Tokyo Bot. Mag. Vol. XXVI. (1912) p. 184.

3) Fr. et Sav. Enum Pl. Jap. Vol. II. p. 463.

4) Salvia japonica Thunb.

5) Matsumura in Tokyo Bot. Mag. Vol. XI. (1897) p. 70. 
"Index Plantarum Japonicarum" editus est; in opere citato, octo species, quattour varietates, quattour formae spontanae et quattuor species introductae nominibus japonicis habitationibusque enumeratae sunt.

Cl. Marino in unodecimo volumine "Tokyo Botanical Magazine"2) plantam japonice "Akinotamuraso" dictam enumeravit. FrancheT et SAVATIER's tres varietates in formas reduxit, et omnes illae formae, sunt in una varietate "typica" dicta contentae. Sicut ipse postea demonstravit, duae species $S$. japonica et chinensis inter se confusae sunt. Demum unam novam varietatem S. japonicae "intermediam" dictam descripsit. Sed haec est ipsa species $S$. japonica, non varietas, quae apud botanicos sub nomine "Natsunotamuraso" nota est. Illius sententia, $S$. nipponica est smilis plantae japonice "Akigiri" dictae; varietas glabrescens hujusdem speciei plantae japonice "Kotojiso" dictae similis est. Anno 1912," in sexto et Vicesimo volumine ejusdem diarii, errorem pristinam correxit. Systema ab illo expositum, est sequens.

Salvia japonica Thuns.

$\alpha$. typica Makino................Natsunotamuraso.

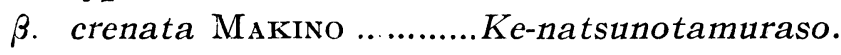

Salvia chinensis BeNTH.

a. typica Makino.

form. a. bipinnata Makino. Akinotamuraso.

form. b. pinnata Makino. Akinotamuraso.

form. c. ternata Makino.

form. d. integrifolia Makino.

\section{ß. pumila Makino. ..............Harunotamuraso.}

Postea, in eodem volumine cl. Makino varietatem "pumilam" ut speciem descripsit, et ei nomen novum ${ }^{4)}$ dedit.

1) Matsumura, Ind. Pl. Jap. II, 2. pp. 547-49.

2) Makino in Tokyo Bot. Mag. Vol. XI (1897) pp. (281-2).

3) Makino, Tokyo Bot. Mag. Vol. XXVI (1912) pp. 78-81.

4) Salvia Ranzaniana, Makino. 
R. Yatabe, olim Professor Universitatis Tokiensis, in libro "Iconograhia Florae Japonicae" anno 1891 edito, unam speciem illustravit. Haec species ab eodem auctore "S. nipponica" esse judicata est, sed ex ejus descriptione et tabula, absque dubio varietas aliqua hujus speciei esse debit.

Ante publicationem Matsumura et Hayata's Enumerationes Plantarum Formosanarum anno 1906 editam, duae species Salviae in insula Formosa notae fuerunt. Prima species, Salvia scapitormis dicta, ab HANCE in "Jounal of Botany" descripta est. Hanc speciem primum endemicam plantam esse putabatur, tamen postea in terra Chinae inventa est. Auctores Forbes et HEMsLey, anno $1890,{ }^{1)}$ in libro "Index Florae Sinensis" dicto, duas species (S. scapiformis et brachiatam) in Formosa inventas enumeraverunt. Henry etiam, in libro "a List of Plants from Formosa" dicto, 2 species enumeravit. Opus systematicum quod nobis multam intelligentiam de genere formosano dedit, est liber Matsumura et Hayata's “Enumerationes Plantarum Formosanarum". Illi auctores duas species adhuc non enumeratas enumeraverunt, et unam novam varietatem "S. scapiformis var. pinnata dictam enumeraverunt. Postea unus illorum auctorum in libro suo "Flora Montana Formosae" dicto, hanc varietatem ad 7702 pedum altitudinem in monte Morrison crescere dixit, et duas novas formas ejusdem speciei (hirsutam et gracilem) descripsit. Species Salviae quae in insula Formosae crescunt, sunt quattuor species, una varietas et duae formae.

Cl. Yokusar Innma, ${ }^{2)}$ in primo volumine ejus libri "SomokuZusetsu" dicti, quattuor sequentes species cum tabulis descriptionibusque illustravit.

1. Salvia nipponica (fol. 27.)............Akigiri. form. argutidens (fol. 28.)............Kotojiso.

2. Salvia japonica (fol. 29.)............Natsunotamuraso.

3. Salvia Ranzaniana(fol. 30.)............Harunotamuraso.

4. Salvia chinensis f. pinnata (fol. 31)..Akinotamuraso.

1) Forbes et Hemsuy, Index Florae Sinensis Vol. II. p. 287.

2) Invuma, Somoku-zusetsu Vol. I. (1832). 
Illi auctores rectam opinionem de duobus speciebus quae diu a multis auctoribus inter se confusae sunt, nobis habuisse viditur.

Tabula sequens docet quo modo species Salviae per singulas insulas Japoniae distribuntur.

\begin{tabular}{l|c|c|c|c|c|c}
\hline$\quad$ Insulae & $\begin{array}{c}\text { Sachalin } \\
\text { Hokkaidô }\end{array}$ & Honsiu & Sikoku & Kiusiu & Liukiu & Formosa \\
\hline Species & & $\times$ & $\times$ & & & - \\
$\begin{array}{l}\text { S. nipponica } \\
\text { var. glabrescens }\end{array}$ & & $\times$ & & & & \\
$\begin{array}{l}\text { S. trisecta } \\
\text { S. miltiorrhiza }\end{array}$ & & & $\times$ & & & \\
S. plectranthoides & & $\times$ & & $\times$ & & $\times$ \\
S. japonica & & & & & \\
S. chinensis & & $\times$ & $\times$ & $\times$ & & \\
S. brachiata & & & & & \\
S. scapiformis \\
$\quad$ var. pinnata & & & & & & $\times$ \\
S. Ranzaniana & & $\times$ & $\times$ & $\times$ & & $\times$ \\
S. pygmaea & & & & & $\times$ & \\
\hline
\end{tabular}

In Honsiu igitur species 5 et una varietas obvisae sunt" quarum nulla endemica est ; Sikoku 5 species habet, e quibus una est endemica; Kiusiu 3; Liukiu 3 (unam endemicam) habet; Formosa 4 species et unam varietatem, habet.

Species sequentes endemicae sunt ; S. nipponica, S. trisecta, S. Ranzaniana, S. pygmaea.

Tabula sequens docet quo modo alterae 6 species per singulas regiones distribuntur.

\begin{tabular}{|c|c|c|c|c|c|c|c|c|c|c|c|c|}
\hline Regiones & 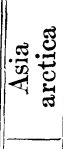 & 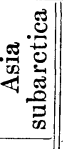 & 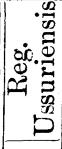 & 岕 & 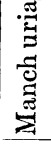 & 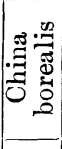 & (3) & 苞 & 芴 & 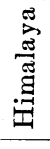 & $\underset{\overparen{Z}}{\overparen{\pi}}$ & 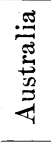 \\
\hline \multirow{7}{*}{$\begin{array}{l}\text { S. miltiorrhiza } \\
\text { S. plectranthoides } \\
\text { S. japonica } \\
\text { S. chinensis } \\
\text { S. brachiata } \\
\text { S. scapiformis }\end{array}$} & & & & & $x$ & & $\times$ & & & & & \\
\hline & & & & & & & & & & $x$ & $x$ & \\
\hline & & & & & & $?$ & $?$ & $?$ & & & & \\
\hline & & & & & & & $\times$ & $\times$ & & & & \\
\hline & & & $\times$ & $x$ & $x$ & $\times$ & $\times$ & $\times$ & $x$ & $\times$ & $\times$ & $x$ \\
\hline & & & & & & & $x$ & & & & & \\
\hline & 0 & 0 & 1 & 1 & 2 & 1 & 4 & 2 & 1 & 2 & 2 & 1 \\
\hline
\end{tabular}


Numerus specierum in insulis japonicis inventarum ad decem increvit. Hae decem species in sequentibus sectionibus ${ }^{1)}$ distribuntur.

1. Sectio Drymosphace Benth.

S. nipponica, trisecta, miltiorhiza.

2. Sectio Notiosphace Bunge.

S. plectranthoides, japonica, chinensis et brachiata.

3. Sectio Gymnosphace BriQ.

S. scapiformis.

4. Sectio Vernales ${ }^{2)}$ Kudo nov. sect.

S. Ranzaniana et pygmaea.

Sectio cujus plantae in insulis japonicis ubique crescunt, est Notiosphace et est ea quae maximum numerum specierum habet. Sectio Gymnosphace subgenus "Allagospodonopsis" quod in systemate generis "Salvia," cum sectione "Allagospodon" est magni monienti, constituit.

1. Salvia nipponica MrQ. Prol. Fl. Jap. p. 39; Fr. et Sav. Enum. Pl. Jap. I. p. 371; Matsum. et Hayata, Enum. Pl. Formos. p. 312; Makino, in Tokyo Bot. Mag. XXI. p. 33; Matsum. Ind. Pl. Jap. II. 2. p. 549; Matsum. et. Kudo, in Tokyo Bot. Mag. XXVI. p. 298.

1) Clavis Sectionum sequens est:

Stamina posteriora ad staminodia reducta. Corollae tubus intus parce

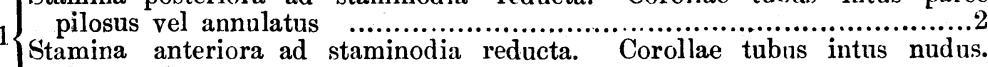
....Gymnosphace.

(Corollae tubus intus distincte annulatus. Flores magni vel parvi ............3 2 Corollae tubus intus parce pilosus non annulatus. Flores minores.......... $2\{$... Vernales.

(Labium corollae superius falciforme. Vectiaria connexa. Bractea et brac$3\left\{\begin{array}{l}\text { teola magnae. Flores saepe magni ............................. Drymosphace. } \\ \text { Labium corollae superium erectum haud falciforme. Vectiaria non connexa. }\end{array}\right.$ Bractea et bracteola minores. Flores saepe parvi............. Notiosphace.

2) Folia omnia vel plerumque radicalia; floralia et bractea minuta. Calyx subtubulosus, labio superiore integro, inferiore bifido. Corollae tubus intus parce pilosus, labium superius bifidum, inferius 4-fidum. Connectiva antice parva, subdeflexa. 
NoM JaP. Akigiri.

HaB. Honsiu, Sikoku et Formosa.

Planta endemica!

form. argutidens Makino, 1. c ; Matsum. 1. c. ; Matsum. et Kudo, 1. c.

Nom. Jap. Kotojiso.

Has. Honsiu.

Planta endemica!

var. glabrescens $F_{R}$. et SAv. Enum. Pl. Jap. I. p. 371 et II. p. 463 ; MaKino, in Tokyo Bot. Mag. XI. p. (282); Matsum. et Kuno, 1. c.

S. glabrescens Makino, in Tokyo Bot. Mag. XXI. p. 157; Matsum. 1. c. p. 547.

S. nipponica YataBE, Icon. Fl. Jap. I. 1, p. 43, t. 15. non MiQ.

Noм. Jap. Miyama-akigiri.

Has. Honsiu.

Planta endemica!

2. Salvia trisecta Matsum. in Matsum. et Kudo, 1. c. p. 298 et Icon. Pl. Koishikav. vol. I. No. 4. p. 125 t. 63.

Nom. Jap. Mitsuba-kotojiso (J. Matsumura).

HAB. Sikoku.

Planta endemica!

3. Salvia miltiorrhiza Bunge, Enum. Pl. Chin. Bor. p. 50 ; Benth. in DC. Prodr. XII. p. 277; Maxim. Mel. Biol. XI. p. 304; Franch. P1. David. p. 236 ; Forb. et Hemsl. Ind. Fl. Sin. II. p. 286 ; Diels, in Engr. Bot. Jahrb. XXIX. p. 557 ; Makino, in Tokyo Bot. Mag. XXII. p. 165.

Nom. Jap. Tanjin.

Hab. Liukiu (ex Makino).

Distrib. China borealis et centralis. 
4. Salvia plectranthoides GrIfF. Notul. IV. p. 199 et Ic. Pl. Asiat. t. 450; Hook. f. F1. Brit. Ind. IV. p. 655 ; Matsum. et Hayata, 1. c. p. 311 ; Matsum. 1. c. p. 549 ; Matsum. et Kudo, 1. c. p. 298 .

Nom. JAP. Hikiokosidamasi. (nov.)

HaB. Formosa.

DisTrib. India, Malaya.

5. Salvia japonica Thunb. Fl. Jap. p. 22 t. 5; Roem. et Schult. Syst. Veg. I. p. 263; Sieb. et Zucc. in Abh. ad. Muench. IV. 3. p. 157; Benth. in DC. Prodr. XII. p. 354; Engl. et Maxim. in ENGL. Bot. Jahrb. VI. p. 66; Makino, in Tokyo Bot. Mag. XXVI. p. 78 ; Matsum. et Kudo, ibidem p. 299.

S. japonica $\beta$. intermedia Makıno in Tokyo Bot. Mag. XI. p. (281), XV. 10 ; Matsum. 1. c. p. 548.

S. japonica $\beta$. intermedia b. lobato-crenata Makino, in Tokyo Bot. Mag. XV. p. 110.

S. japonica $\gamma$. bipinnata FR. et SAv. Enum. P1. Jap. I. p. 372, II. p. 463. (p. p.)

Nom. JAP. Natsunotamuraso.

Hab. Honsiu et Kiusiu.

Distrib. China (?).

form. pinnata Matsum et Kudo, 1. c. foliis pinnatis.

Nom. Jap. Haneba-no-natsunotamuraso. (nov.).

HaB. Honsiu.

Planta endemica!

form. ternata Matsum. et Kudo, 1. c. foliis ternatis.

Nom. JAP. Mitsuba-no-natsunotamuraso.

HaB. Honsiu.

Planta endemica!

var. crenata Makino in Tokyo Bot. Mag. XXVI. p. 79.

S. japonica $\beta$. intermedia a. crenata Makino in Tokyo Bot. Mag. XV. p. 110.

Nom. Jap. Ke-natsunotamuraso.

$\mathrm{H}_{\mathrm{AB}}$. Honsiu.

Planta endemica! 
6. Salvia chinensis Benth. Lab. Gen. et Spec. p. 725 et in DC. Prodr. XII. p. 355 ; Makino in Tokyo Bot. Mag. XXVI. p. 80; Matsum. et Kudo, 1. c. p. 299.

$S$. japonica $\beta$. integrifolia Fr. et SAv. Enum. Pl. Jap. I. p. 371 et II. p. 463; Forb. et HemsL. Ind. Fl. Sin. II. p. 284; Diels, in Engl. Bot. Jahrb. XXIX. p. 558.

S. japonica a. typica c. integrifolia MAKINo in Tokyo Bot. Mag. II. p. (281) et XV. p. 108 ; Matsum. 1. c. p. 548.

S. chinensis $\alpha$. typica d. integrifolia Makino, in Tokyo Bot. Mag. XXVI. p. 80.

NoM. JAP. Maruba-no-akinotamuraso.

Hab. Honsiti.

Distrib. China borealis et centralis.

form. b. Fortunei Matsum. et Kudo, 1. c. p. 299.

S. Fortunei Benth. in DC. Prodr. XII. p. 354 et Fl. Hongk. p. 277 ; Brig. in ENGL. Nat. Pfl.fam. IV. 3a p. 285.

S. diversifolia Mro. Prol. F1. Jap. p. 40.

S. japonica $\beta$. ternata Fr. et SAv. Enum. P1. Jap. I. p. 372 et II. p. 463 ; FR. P1. David. I. p. 236 ; Diels, in EngL. Bot. Jahrb. XXIX. p. 558.

S. japonica $\alpha$ typica b. ternata Makino, in Tokyo Bot. Mag. II. p. (281) et XV. p. 108 ; Matsum. 1. c. p. 548.

S. chinensis o. typica c. ternata Makino, in Tokyo Bot. Mag. XXVI. p. 80.

Nom. Jap. Mitsuba-no-akinotamuraso, Mitsuba-no-tamuraso.

HAB. Honsiu.

Distrib. China.

form. c. pinnata (Diels) Matsum. et Kudo, 1. c. p. 299.

S. japonica var. pinnata Drels in Engl. Bot. Jahrb. XXIX. p. 558.

S. chinensis $\alpha$. typica b. pinnata Makino, in Tokyo Bot. Mag. XXVI. p. 80.

Nom. JAP. Akinotamuraso, Haneba-no-Akinotamuraso.

HaB. Honsiu.

Distrib. China. 
form. d. bipinnata MaKINo, in Tokyo Bot. Mag. XXVI. p. 80; Matsum. et Kudo, 1. c.

S. japonica bipinnata Fr. et SAv. Enum. Pl. Jap. I. p. 372 et II. p. 463. p. p.

S. japonica a. tpyica a. bipinnata MAKINo, in Tokyo Bot. Mag. XI. p. (281) et XV. p. 107; Matsum. 1. c.

Nom. JaP. Akinotamuraso.

Hab. Honsiu.

Distrib. China.

form. e. alatopinnata Matsum. et Kudo, 1. c. foliis pinnatis vel bipinnatis, pinnis lanceolatis basi cuneatis.

Nom. Jap. Togakushi-akinotamuraso.

Hab. Honsiu.

Planta endemica!

7. Salvia brachiata Roxв. Hort. Beng. p. 4 et Fl. Ind. I. p. 145 ; Diels in EngL. Bot. Jahrb. XXIX. p. 558 ; Matsüm. et Kudo, 1. c. p. 299.

S. plebeia R. Br. Prodr. Fl. Nov. Holl. p. 501; Benth, in DC. Prodr. XII. p. 355, et Fl. Hongk. p. 277; MiQ. Prol. F1. Jap. p. 40 ; Maxim. Ind. Fl. Pek. in Prim. Fl. Amur. p. 475 ; BAKER et Moore, in Jour. Linn. Soc. XVII. p. 385 ; Fr. et SAy. Enum. Pl. Jap. I. p. 372 ; Fr. Pl. David. p. 237 ; Forb. et Hemsl. Ind. Fl. Sin. II. p. 287 ; Palib. Consp. Fl. Kor. II. p. 28 ; Kom. Fl. Mansh. III. p. 373; Matsum. et Hayata, Enum. Pl. Formos. p. 312 ; Matsum. 1. c. p. 549 ; Nakai, Fl. Kor. II. p. 141.

NoM. JAP. Yukimiso.

Hab. Honsiu, Kiusiu, Liukiu, Formosa.

Distrib. China, Manchuria, Corea, India, Malaya, Australia.

8. Salvia scapiformis $\mathrm{H}_{\mathrm{ANCE}}$, in Jour. Bot. (1885) p. 368; Bot. Mag. t. 6980; Forb. et HemsL. Ind. Fl. Sin. II. p. 287; Henry List Pl. Formos. p. 73; Diels, in Engl. Bot. Jahrb. XXIX. p. 559 ; Matsum. et Hayata, 1. c. p. 312 ; Hayata, Fl. Mont. Formos. p. 182 ; Matsum. et Kudo, 1. c. p. 299. 
NoM. Jap. Takasago-tamuraso. (nov.)

Distrib. China centralis.

var. pinnata Hayata, in Matsum. et Hayata, 1. c. p. 312 t. 17 ; Hayata, Fl. Mont. Formos. p. 182 ; Matsum. et Kudo, 1. c.

Hab. Formosa.

Planta endemica!

form. hirsuta Hayata, Fl. Mont. Formos. p. 182 ; Matsum. et KuDo, 1. c.

Hab. Formosa.

Planta endemica!

form. gracilis Hayata, 1. c. p. 183 ; Matsum. et Kudo, 1. c. Hab. Formosa.

Planta endemica!

9. Salvia Ranzaniana Makino, Tokyo Bot. Mag. XXVI. p. 184 ; Matsum. et Kudo, 1. c. p. 299.

S. japonica o. pumila Fr. et SAv. Enum. Pl. Jap. I. p. 372 et II. p. 463; Makino, in Tokyo Bot. Mag. XI. p. (281) et XV p. 110 ; Matsum. 1. c.

S. chinensis var pumila Makino ibidem XXVI. p. 81.

S. vernalis Matsum. ET Kudo, in Sched. Herb. Sc. Coll. Imp. Univ. Tokyo.

Nom. JaP. Harunotamuraso.

HaB. Honsiu, Sikoku, Kiusiu.

Planta endemica!

10. Salvia pygmaea Mastum. in Tokyo Bot. Mag. II. p. 70 et Ind. P1. Jap. II. 2. p. 549 ; Matsum. et Kudo, 1. c. p. 299 ; Makino, ibidem. p. 212.

Nom. Jap. Himetamuraso.

Haв. Liukiu.

Planta endemica! 